\title{
VSOP OBSERVATIONS OF THE BL LAC OBJECT 2007+777 *
}

\author{
C. JIN \\ Beijing Astronomical Observatory, National Astronomical Observatories, China \\ T.P. KRICHBAUM and A. WITZEL \\ Max-Planck-Institut für Radioastronomie, Germany \\ R. NAN and B. PENG \\ Beijing Astronomical Observatory, National Astronomical Observatories, China
}

A. KRAUS, A. LOBANOV, S. QIAN and J.A. ZENSUS

Max-Planck-Institut für Radioastronomie, Germany

\begin{abstract}
We present three epochs of VSOP observations of the BL Lac object $2007+777$ at $5 \mathrm{GHz}$. Compared with the ground-based VLBA data, the space baselines with HALCA clearly reveal a more detailed and finer source structure. Mainly based on the quite uniform and circular UV-coverages of the VLBA, and using a new cross-selfcalibration method, we have found evidence for weak structural changes on a timescale of two weeks in the core region of this intraday variable source. The physical causes for these variations are discussed.
\end{abstract}

Keywords: BL Lac, IDV, structural variability

\section{Introduction}

The superluminal radio source $2007+777$ (Witzel et al., 1988) was identified as a BL Lac object owing to its almost featureless optical spectrum (Biermann et al., 1981). It recently showed rapid variations on time scales of days at optical, infrared and radio wavelengths (Peng et al., 2000). Flux density measurements with the $100 \mathrm{~m}$ radio telescope at Effelsberg show that $2007+777$ belongs to the slower class of intraday variable radio sources (type I). In this paper, we present VSOP observations of the source at 3 epochs, which were spaced by 4 days, 2 and 3 weeks.

\section{Observations and Data Reduction}

The VSOP observations were performed in 1998 at $5 \mathrm{GHz}$, see Table I. The data were correlated at NRAO, Socorro. Because of the expected large fringe delay offset for HALCA (the fringe fitting for HALCA removed indeed a large residual

* This research was partially supported by a grant from the National Natural Science Foundation of China. 
TABLE I

The experiments, where TZ, GZ and RZ are the tracking stations for HALCA

\begin{tabular}{lll}
\hline epoch & date & antennas \\
\hline 1 & 20-MAR-1998 & BR,FD,HN,KP,LA,MK,NL,OV,PT,SC,Y, HALCA(TZ) \\
2 & 01-APR-1998 & BR,FD,HN,LA,MK,NL,OV,KP,PT,SC,Y, HALCA(failed) \\
3 & 05-APR-1998 & BR,FD,HN,LA,MK,NL,SC, HALCA(RZ,GZ,TZ) \\
\hline
\end{tabular}
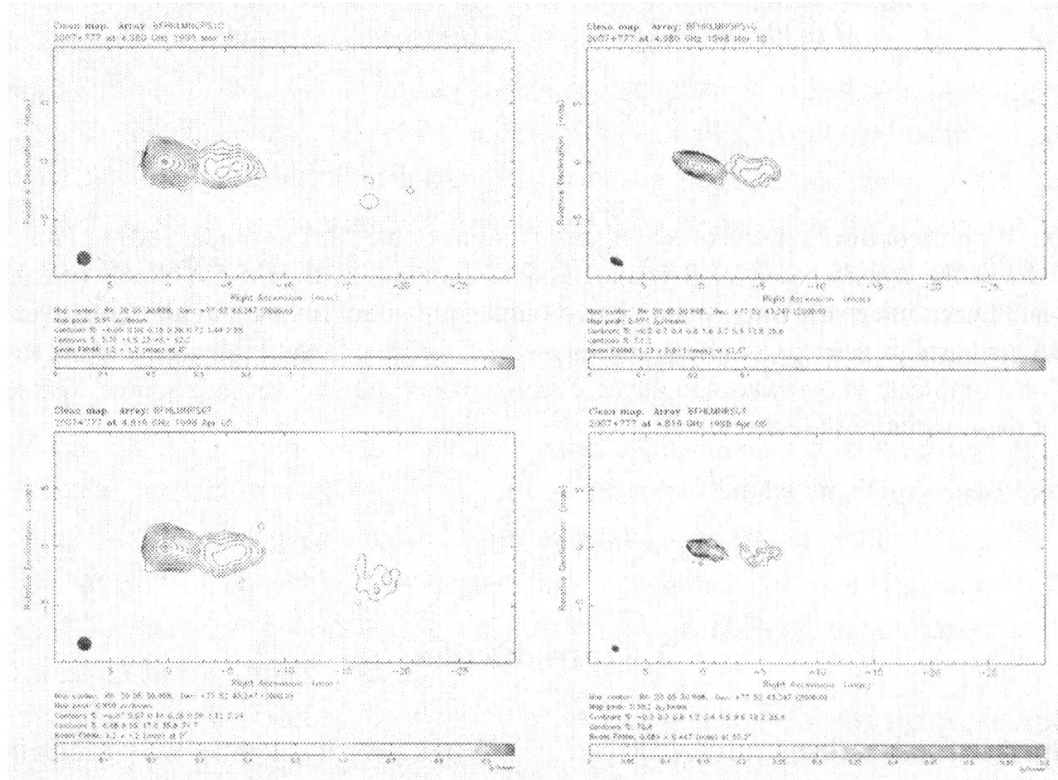

Figure 1. We show the ground-based VLBI images (left) and the VLBI images including HALCA (right). The panels show the first (top) and the third (bottom) epochs.

delay of a few hundred nano-seconds), the data were correlated with 256 channels in one $16 \mathrm{MHz}$ band. This avoids bandwidth smearing. Clear fringes were found to HALCA, except for the second epoch. Calibration and fringe fitting was done in AIPS (following Chapter 9 of the AIPS Cookbook). The data were exported using SPLIT. The hybrid mapping was done in DIFMAP.

\section{Results and Discussion}

The contour maps of the BL Lac object $2007+777$ are shown in Figure 1. We only present maps of the first and the last epoch, since at the second epoch HALCA failed. 

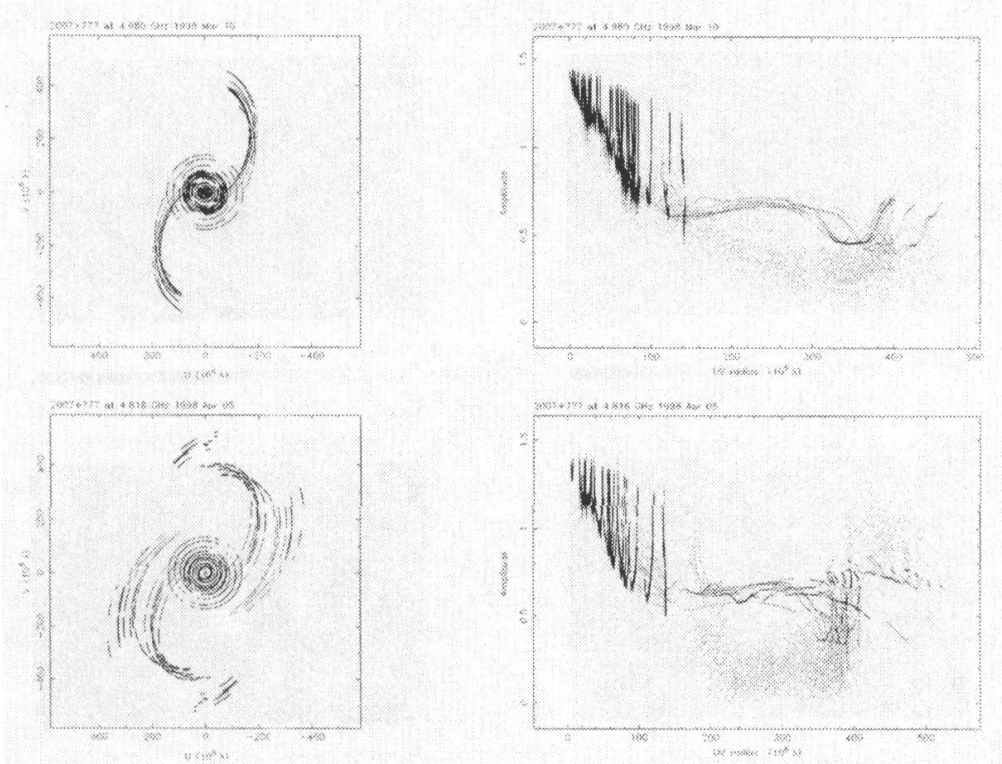

Figure 2. The UV-coverages and all the UV-data of the first (top) and the third (bottom) epoch. Lines indicate a clean component model obtained from the ground-array alone. It is seen that the HALCA baselines show additional structure.

The ground array shows an east-west oriented core-jet structure of $\sim 20$ mas site. With the higher resolution of the space baselines, more details and a finer structure becomes visible, particularly in the vicinity of the core (at $r \leq 2$ mas). That there is indeed structure on HALCA baselines, which cannot be seen with the ground array, becomes obvious when inspecting Figure 2. The Figure shows large deviations between the HALCA data and clean components obtained from the ground-array alone. This suggests that the source has a rich morphology also below the milli-arcsecond scale. Of course, better maps of higher dynamic range and a more uniform UV-coverage will be required for the future.

The richness of the source structure on milli-arcsecond scales makes it difficult to confirm structural changes solely from Gaussian model fits to the data. Thus, we tried a new method in order to compare the closure quantities of the data from the different epochs: using the final clean components from an arbitrary epoch as input model, we selfcalibrated the data from the other epoch and inspected the residual maps (see Figure 3). In this approach, the clean components act as the carrier of the closure quantities. After the data and the model are exchanged, a peak of about $3.0-4.0 \mathrm{mJy}$ appears in the residual maps. This deviation is about 10 times larger than the RMS in the images calibrated on themselves. This suggests that some structural variations in the core region of the source have occurred. At present, we are still investigating whether the differences in the UV-coverages could cause such 


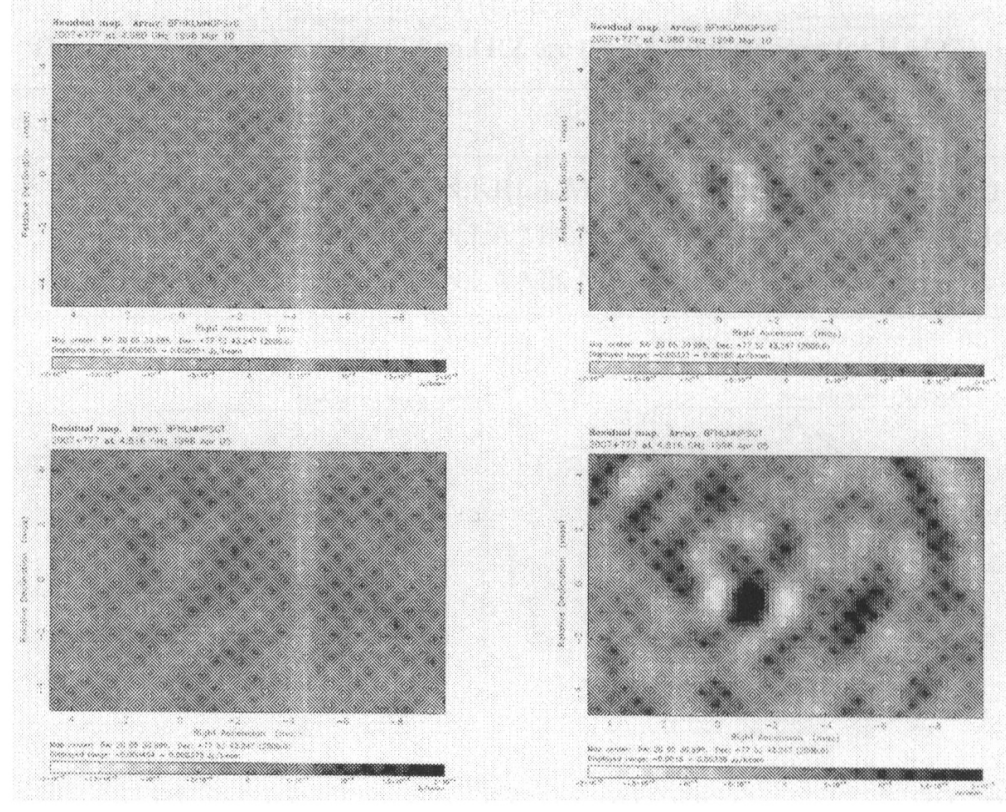

Figure 3. Comparison of the residual maps from the first (top) and the third (bottom) epochs. The maps on the left show the residuals after self-calibration with the model obtained at this epoch. On the right the residual maps are shown after self-calibration with the model from the other epoch. The cross-wise exchange reveals clear differences in the residual noise pattern.

an effect. Future observations with a more uniform UV-coverage at all observing epochs will be required to confirm our still tentative findings.

The detection of an unresolved (not scatter broadened) compact core with a size much smaller than the typical galactic scattering size of $0.5-2$ mas argues against time dependent image distortions due to propagation effects (RISS). Atmospheric effects can be ruled out, since antenna-dependent amplitude and phase errors are removed by the self-calibration technique. The time scale of the structural variations is of order of $2-3$ weeks, similar to the variations seen in the total flux density. This and the fact that the structural changes appear near or in the VLBI core, lead us suspect that they are intrinsic to the source and not due to interstellar scintillation.

\section{References}

Biermann, P., Duerbeck, H., Eckart, A., et al.: 1981, ApJ 247, L53.

Peng, B., Kraus, A., Krichbaum, T.P., et al.: 2000, A\&A 353, 937.

Witzel, A., Schalinski, C.J., Johnston, K.J., et al.: 1988, A\&A 206, 245. 